\title{
Analysis of Losses in Cables and Transformers with Unbalanced Load and Current Harmonics
}

\author{
Yunxia Dong*, Yuefeng Shi \\ College of Electrical and Electronic Engineering, North China Electric Power University, Beijing, China \\ Email address: \\ dyx2007@126.com (Yunxia Dong) \\ ${ }^{*}$ Corresponding author \\ To cite this article: \\ Yunxia Dong, Yuefeng Shi. Analysis of Losses in Cables and Transformers with Unbalanced Load and Current Harmonics. Journal of \\ Electrical and Electronic Engineering. Vol. 9, No. 3, 2021, pp. 78-86. doi: 10.11648/j.jeee.20210903.13
}

Received: April 17, 2021; Accepted: May 25, 2021; Published: June 4, 2021

\begin{abstract}
The power quality problems are more and more serious in the power system. The harmonics and the three-phase imbalance have a greater impact on the line loss of power grid. We focus on the power losses in cables and transformers with unbalanced load in current harmonics basing on the equivalent resistance method. The impact caused by the skin effect is illustrated in order to enhance the accuracy of the theoretical calculation. For this purpose, a mathematical development that takes in account the presence of current harmonic components and unbalanced load in the losses calculation is presented. The reliability of the method is verified in a simulation model. According to the comparative analysis between the simulation and the proposed method, we can conclude that the harmonic resistance calculation method considering skin effect is more accurate than the traditional method. The results show that the additional loss caused by the harmonics has a nonlinear relation with the harmonic currents. At the same time, the additional loss of distribution network under compound disturbance with unbalanced load in current harmonics is studied. Compared with the cases that two phase load are heavy and one phase load is light or one phase load is heavy and other two phase load are light, when one phase load is heavy, one phase load is light and one phase load is the average value of the others, the additional loss of the system is the least one. The additional loss for the case with that two phase load are heavy and one phase load is light is the biggest one.
\end{abstract}

Keywords: Power Losses, Skin Effect, Harmonics, Unbalanced Load

\section{Introduction}

With the continuous improvement of economic level, the dependence of people's production and life on power is also growing [1]. At the same time, higher requirements are put forward for power quality. For the three-phase four-wire low-voltage distribution network to be studied in this paper, the power quality problem is mainly caused by the application of a large number of nonlinear power electronic loads. Among the usual power quality problems, the harmonics and the three-phase imbalance have a greater impact on the line loss of power grid [2]. In view of the above situation, a large number of scholars put forward their own opinions on the loss calculation and management of low voltage distribution network under harmonic and three-phase unbalanced disturbances. The author derived the calculation method of theoretical line loss under unbalanced phase angle and amplitude in the reference [3]. Reference [4] showed the study of the low-voltage distribution network under the composite disturbance of harmonic and three-phase unbalance, and verified the proposed decoupling idea for composite power quality disturbance through theoretical analysis and simulation. Reference [5] compared and analyzed the line loss of distribution network under composite power quality disturbance with that under single power quality disturbance, and proposed that three-phase aggravated the harmonic loss. Reference [6] studied the loss of lines and transformers under harmonic disturbance from an economic point of view, and proposed that the line loss caused by harmonic disturbance must be compensated. Reference [7] compared and analyzed the harmonic loss of distribution network under three-phase unbalanced and three-phase balanced conditions, and proposed that under three-phase unbalanced conditions, the increase of harmonic loss mainly came from the neutral line. The harmonic currents due to electric vehicles charging are studied [8]. The extensive employment of nonlinear loads and 
large-scale grid connected distributed grid units has led to considerably distorted voltages and currents in the distribution systems $[9,10]$. Accordingly, in the recent studies [11, 12], the harmonic distortion limits have been considered. The calculation method of the additional losses of the transmission line and the transformer in three-phase imbalance is derived theoretically, and the loss caused by the harmonic current is not considered [13].

Based on the above analysis, the analysis of harmonic loss of distribution network is mainly based on the equivalent resistance method, the main problem is that it only considers the fundamental wave and ignores the influence of skin effect on harmonic resistance under nonlinear load. The research in references $[14,15]$ showed that when harmonics exist, the increase of line resistance caused by skin effect has a great influence on line loss. To sum up, after studying the influence of skin effect on line resistance, we propose a calculation method to get the harmonic resistance, and verify that the calculation method has high reliability under single power quality disturbance and composite power quality disturbance. Based on the method in this paper, the additional loss of the distribution network under the composite power quality disturbance such as the unbalanced load and the current harmonics can be accurately calculated. The calculated results are verified by the simulation.

\section{Calculation and Analysis of Losses of Transformer and Cable Under Harmonic Currents}

\subsection{Derivation of Conductor Resistance Under Harmonic Currents}

Usually, a conductor can be represented as a pure resistance which consumes the electricity energy, as shown in Figure 1. The losses due to harmonic currents are basically proportional to the square of the current. However, one should also consider the resistance increase with the frequency due to the skin effects of conductors. In order to study the variation mechanism of harmonic resistance, it is first necessary to study the distribution of current density in the conductor [16].

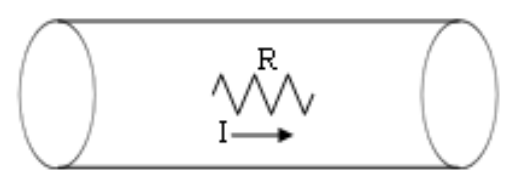

Figure 1. Current flowing through a cable.

For the electromagnetic fields that in conductors with low frequency currents, the internal electromagnetic fields change slowly with time, so the effect of the rate of change of electric flux density in Maxwell's equations is ignored, and the Maxwell's equations are simplified. Therefore, we have

$$
\left\{\begin{array}{l}
\nabla \times H=\gamma E \\
\nabla \cdot B=0 \\
\nabla \times E=-\frac{\partial B}{\partial t} \\
\nabla \cdot D=0
\end{array}\right.
$$

where $\mathrm{H}$ is magnetic field intensity, $\gamma$ is conductivity, $\mathrm{E}$ is electric field intensity, $\mathrm{B}$ is magnetic flux density and $\mathrm{D}$ is electric displacement.

It can be obtained from the equations above,

$$
\nabla^{2} E=\mu \gamma \frac{\partial E}{\partial t}
$$

in which $\mu$ is permeability of vacuum. Its plural form is

$$
\nabla^{2} \dot{E}=j \omega \omega \gamma \dot{\bar{E}}
$$

Here $\omega$ is angular frequency electromagnetic wave.

Assume that $m=\sqrt{j \omega \mu \gamma}=\sqrt{\frac{\omega \mu \gamma}{2}}(1+j)=\frac{1}{\delta_{h}}(1+j)$, in which $\delta_{h}$ is skin depth under the harmonic whose order is $h$; $\delta_{h}=\sqrt{\frac{2}{\omega \mu h}}=\sqrt{\frac{2}{h \omega_{0} \mu \gamma}} \cdot \omega_{0}$ is the angular frequency of the fundamental wave. Then the equation above could be written as $\nabla^{2} \dot{E}=m^{2} \dot{E}$.

We can solve from the equations above that

$$
\dot{E}(r)=A e^{-m r}+B e^{m r}
$$

Assume that the radius of the circular conductor is $r_{0}$ and the direction of current is perpendicular to the cross section of circular conductor as shown in Figure 1. Then the current density function is only related to the conductor radius and it could be written as $J(r)$. Because $J(r)=\gamma E$, therefore, electric field intensity is also a function of conductor radius, could be written as $E(r)$.

Establish boundary conditions: the electric field intensity of conductor surface is $\dot{E}_{0}$, while $\dot{E}\left(r_{0}\right)=\dot{E}\left(-r_{0}\right)=\dot{E}_{0}$, the following equation will be obtained after we substitute it into the equation above:

$$
\dot{E}(r)=\frac{e^{-m r}+e^{m r}}{e^{-m r_{0}}+e^{m r_{0}}} \dot{E}_{0}
$$

Because $\dot{J}_{0}=\gamma E_{0}$, then the current density function in the conductor is

$$
\dot{J}(r)=\gamma \dot{E}(r)=\frac{e^{-m r}+e^{m r}}{e^{-m r_{0}}+e^{m r_{0}}} J_{0}
$$


By calculating the complex modulus, the current density amplitude can be obtained

$$
J(r)=J_{0} \sqrt{\frac{\frac{e^{-\frac{2 r}{\delta h}}+e^{\frac{2 r}{\delta h}}}{2}+\cos \left(\frac{2 r}{\delta h}\right)}{\frac{e^{-\frac{2 r_{0}}{\delta h}}+e^{\frac{2 r_{0}}{\delta h}}}{2}+\cos \left(\frac{2 r_{0}}{\delta h}\right)}}
$$

As the equation above is too complex for practical application, it needs to be reasonably simplified. Suppose the conductor is a semi-infinite plane conductor, $r_{0} \rightarrow \infty$, $0<e^{-\frac{2 r_{0}}{\delta_{h}}}<1,-1 \leq \cos \left(\frac{2 r_{0}}{\delta_{h}}\right) \leq 1$, it can be ignored when compared with $e^{\frac{2 r_{0}}{\delta_{h}}}$. Moreover, when $r$ is small enough, $J(r) \approx 0$, which means we can ignore the influence of $e^{-\frac{2 r}{\delta_{h}}}$ and $\cos \left(\frac{2 r}{\delta_{h}}\right)$. Therefore, the current density amplitude of the conductor can be obtained by simplified calculation as

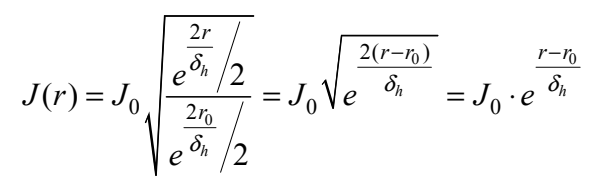

If the conductor is treated as a cylinder, the current passing through it is

$$
\begin{aligned}
I & =\int_{r}^{r_{0}} J(r) \cdot 2 \pi r d r \\
& =2 \pi J_{0}\left[r_{0} \delta_{h}+\delta_{h}^{2} e^{-\frac{r_{0}}{\delta h}}-\delta_{h}^{2}\right]
\end{aligned}
$$

The current density of the harmonic on the surface of the conductor can be obtained as

$$
J_{0}=\frac{I}{2 \pi\left[r_{0} \delta_{h}+\delta_{h}^{2} e^{-\frac{r_{0}}{\delta h}}-\delta_{h}^{2}\right]}
$$

The thermal power generated per unit length of wire can be written as

$$
\begin{aligned}
P & =\int_{r}^{r_{0}} \rho \cdot J^{2}(r) \cdot 2 \pi r d r \\
& =\int_{r}^{r_{0}} \rho \cdot J_{0}^{2} \cdot e^{\frac{2\left(r-r_{0}\right)}{\delta_{h}}} \cdot 2 \pi r d r \\
& =\pi J_{0}^{2}\left[\rho\left(r_{0} \delta_{h}-\frac{\delta_{h}^{2}}{2}+\frac{\delta_{h}^{2}}{2} e^{-\frac{2 r_{0}}{\delta_{h}}}\right)\right]
\end{aligned}
$$

where $\rho$ is resistivity of the conductor.

The unit length resistance of conductor under harmonic is:

$$
R_{\mathrm{h}}=\frac{P}{I^{2}}=\frac{\rho \delta_{h}}{4 \pi} \cdot \frac{\left(\mathrm{R}_{0}+\frac{\delta_{\mathrm{h}}}{2} \mathrm{e}^{-\frac{2 R_{0}}{\delta_{h}}}-\frac{\delta_{h}}{2}\right)}{\left(\mathrm{R}_{0} \delta_{h}+\delta_{h}^{2} \mathrm{e}^{-\frac{R_{0}}{\delta_{h}}}-\delta_{h}^{2}\right)}
$$

In order to represent the harmonic resistance values conveniently under different harmonics, the amplification coefficient of harmonic resistance has been proposed as $k_{h}$.

$$
k_{\mathrm{h}}=\frac{R_{h}}{R_{1}}
$$

where $R_{1}$ is resistance value of unit length conductor under fundamental current.

Therefore, the harmonic resistance calculation formula is

$$
R_{\mathrm{h}}=L k_{h} R_{1}
$$

where $R_{\mathrm{h}}$ is the resistance value of full-length conductor under the harmonic wave while $h$ is the order of harmonic current; $L$ is the length of the wire.

\subsection{Additional Losses of Transformer and Cable Under Harmonics}

Since the influence of power quality disturbance on transformer iron loss is relatively small and can be ignored, we only study the additional copper loss of transformer. Therefore, the equivalent circuit of low voltage distribution network can be expressed in Figure 2 below.

Since the low voltage distribution network contains multiple harmonic, according to the derivation above, the additional loss calculation formula of cable and transformer under harmonic disturbance can be obtained respectively:

$$
\Delta P_{H, T}=\Delta P_{H, P L}+\Delta P_{H, N L}=3 \sum_{h=2}^{\infty} I_{h, P L}^{2} R_{h, P L}+\sum_{h=2}^{\infty} I_{h, N L}^{2} R_{h, N L}
$$

where $R_{\mathrm{h}, P L}$ is the equivalent phase cable resistance under $h$ order harmonics; $R_{h, N L}$ is the equivalent neutral cable resistance under harmonic; $I_{h, P L}$ is the phase cable harmonic currents; $I_{h, N L}$ is the harmonic current of neutral cable.

$$
\Delta P_{H, T}=3 \sum_{h=2}^{\infty} I_{h}^{2} R_{h, T}
$$

where $R_{h, T}$ is the equivalent resistance under harmonic of transformer winding and $I_{h}$ is the harmonic currents flowing through the transformer. The total additional loss caused by the harmonics can be written as

$$
\begin{aligned}
\Delta P_{H} & =\Delta P_{H, L}+\Delta P_{H, T} \\
& =3 \sum_{h=2}^{\infty} I_{h, P L}^{2} R_{h, P L}+\sum_{h=2}^{\infty} I_{h, N L}^{2} R_{h, N L}+3 \sum_{h=2}^{\infty} I_{h}^{2} R_{h, T}
\end{aligned}
$$




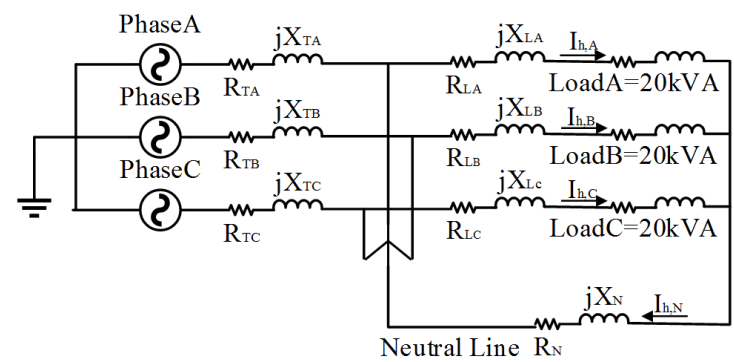

Figure 2. Equivalent model of low-voltage distribution network under power quality disturbance.

\subsection{Simulation Verification and Comparative Analysis}

The basic idea of traditional equivalent resistance method is to assume an equivalent line resistance $\mathrm{R}_{\mathrm{eq}}$ at the beginning of distribution line. Thus, the total loss of the distribution network is caused by the equivalent resistance and the total current flowing into the distribution network.

Table 1. System parameters of the simulation model.

\begin{tabular}{ll}
\hline system elements & parameters \\
\hline Rated voltage of voltage source & $10 \mathrm{kV}$ \\
Rated capacity of voltage source & $25 \mathrm{MVA}$ \\
Transformer rated capacity & $315 \mathrm{kVA}$ \\
Transformer resistance unit value & $0.2 \mathrm{p} . \mathrm{u}$ \\
Cable and neutral cable resistance & $1 \Omega$ \\
Rated voltage with load & $220 \mathrm{~V}$ \\
Load rated capacity & $20 \mathrm{kVA}$ \\
\hline
\end{tabular}

In order to verify the feasibility of the harmonic resistance calculation method based on the principle of engineering electromagnetic field derived in this paper, and to study the additional loss characteristics of transformer and cable under harmonic disturbance, a three-phase four-wire low-voltage distribution network model is built in MATLAB software for simulation verification and comparative analysis.

When the harmonic current is injected into the load side of the model, and additional losses due to harmonic disturbance will be generated on the cables and the transformer. We consider there are just third and fifth harmonic currents except the fundamental current in the system. According to the definition that $T H D=\sqrt{\sum_{h=2}^{\infty}\left(I_{h} / I_{1}\right)^{2}}$, the total harmonic distortion rate is the ratio of the mean square root of all harmonic content to the mean square root of fundamental wave. Therefore, we can use the total harmonic distortion rate to characterize the change of harmonic content in the system. By controlling the variables, only the total harmonic distortion rate of the system is changed. The curves of the additional loss of the system obtained by simulation and two calculation methods with the total harmonic distortion rate are recorded respectively. The value obtained by simulation is compared with the theoretical value of additional loss obtained by the harmonic resistance calculation method proposed in this paper and the theoretical value of additional loss obtained by the equivalent resistance method. The results for additional losses of transformers and cables are shown in Figures 3 and 4 respectively. Figure 4 shows the additional losses of transformers under third and fifth harmonic currents using simulation result. According to the comparative analysis of the graphical results, it can be verified that under the same conditions, the results obtained by the method proposed in this paper are closer to the simulation results, indicating that the method proposed in this paper has higher reliability.

In order to further study the influence of each harmonic on the additional loss of distribution network under harmonic disturbance, maintain three-phase load balance and contain only three or five harmonics, additional loss of low voltage distribution network are recorded as shown in Figure 5. According to the definition that $H R I_{h}=\sqrt{\left(I_{h} / I_{1}\right)^{2}}$, the harmonic content rate refers to the ratio of the mean square root value of the harmonic current to the mean square root value of the fundamental current. Therefore, the harmonic content rate of the third or fifth gradually changed, that is, the size of the harmonic current flowing through the system is changed. The additional losses of low-voltage distribution network caused by third and fifth harmonics under the same harmonic content are compared. According to the results shown in the Figure 5, the higher the harmonic content rate, the greater the total additional loss generated in the distribution network. Meanwhile, the higher the harmonic frequency is, the greater the additional loss is. It is further verified that the skin effect caused by higher harmonics will increase the cable impedance.

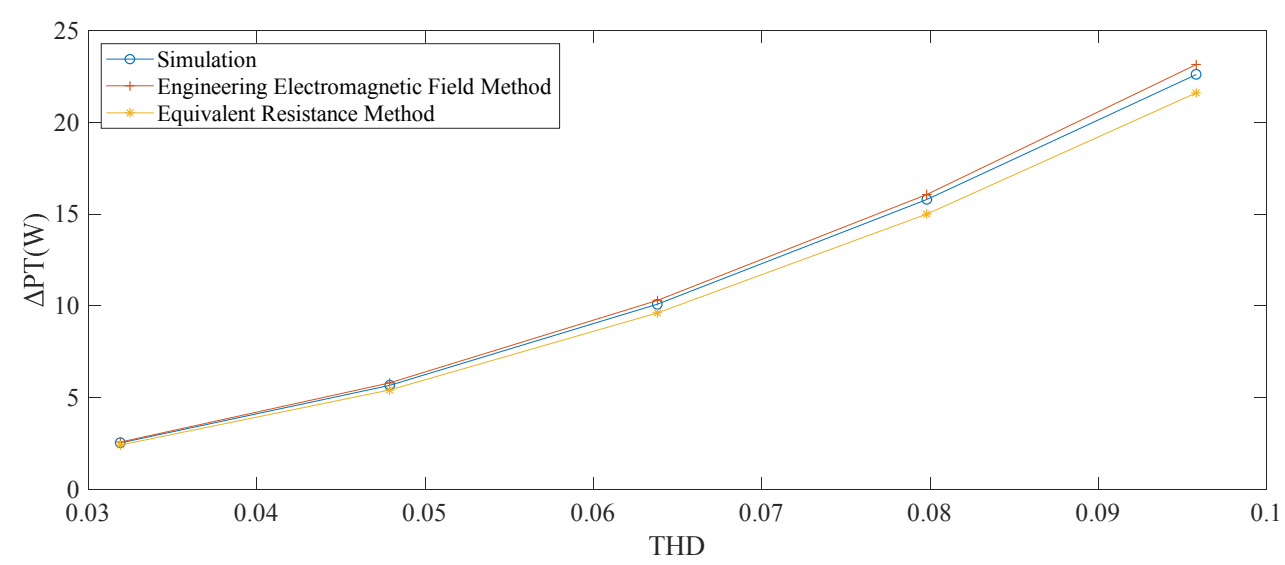

Figure 3. Comparison of additional losses of transformers under third and fifth harmonic disturbances. 


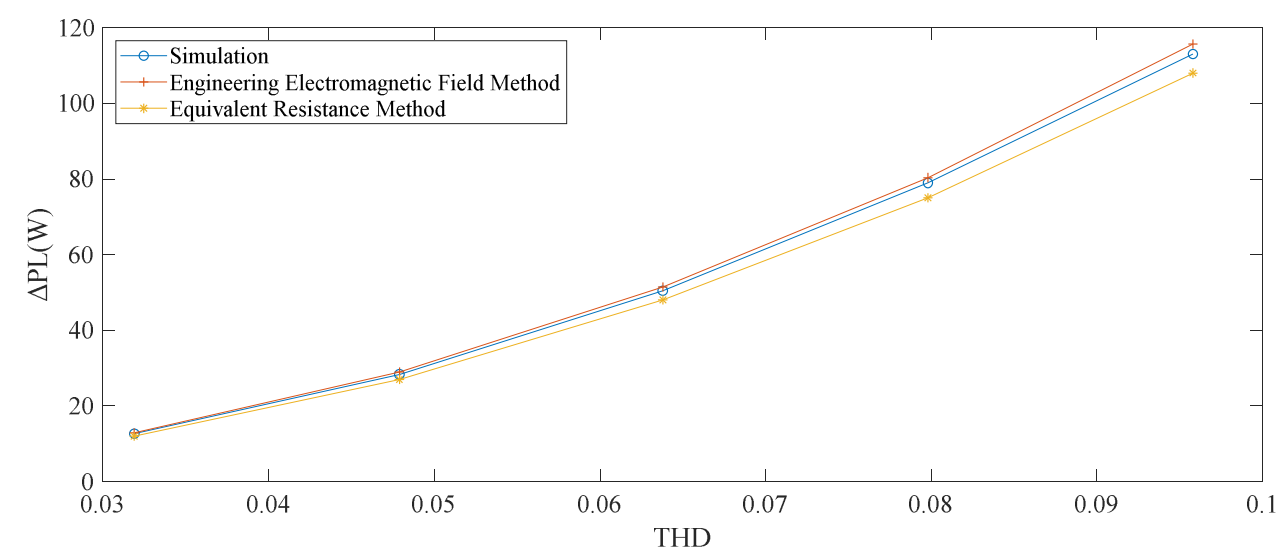

Figure 4. Comparison of additional losses of cable under 3 and 5 harmonic disturbances.

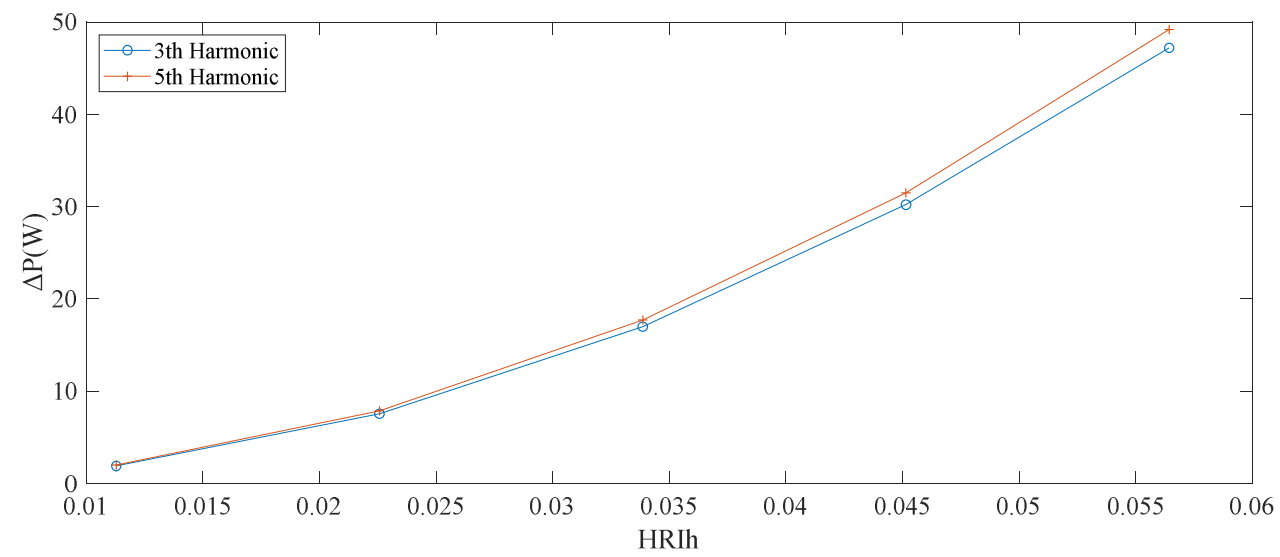

Figure 5. Analysis of influence of third and fifth harmonics on additional loss of distribution network under harmonic disturbance.

\section{Simulation Verification and Analysis of Losses of Cable and Transformer Under Composite Disturbance}

\subsection{Calculation Method of Additional Losses of Cable and Transformer Under Composite Disturbance}

In order to verify the general applicability of the proposed harmonic resistance calculation method, it is necessary to verify that it is still reliable under various complex disturbances. Therefore, the calculation formula of the additional loss of the distribution network under the composite power quality disturbance with harmonic and three-phase imbalance is derived. Three-phase unbalance here mainly refers to three-phase load unbalance.

When there is no harmonic flow in the low-voltage distribution network and the three-phase load is balanced, the cable loss is as follows:

$$
P_{B, L}=3 I_{B, P L}^{2} R_{1, P L}
$$

where $I_{B, P L}$ is three-phase balanced phase cable current value and $R_{F, P L}$ is phase cable resistance under fundamental wave.

In case of three-phase unbalance, the loss on the cable is:

$$
\begin{aligned}
P_{U B, P L} & =\left[\left(1+\beta_{\alpha}\right)^{2}+\left(1+\beta_{\mathrm{b}}\right)^{2}+\left(1+\beta_{c}\right)^{2}\right] I_{a v}^{2} R_{F, P L} \\
& =\left(\beta_{a}^{2}+\beta_{b}^{2}+\beta_{c}^{2}+3\right) I_{a v}^{2} R_{F, P L}
\end{aligned}
$$

Of which,

$$
\left\{\begin{array}{l}
\dot{I}_{a}=I_{a v}\left(1+\beta_{a}\right) \angle \phi_{a} \\
\dot{I}_{b}=I_{a v}\left(1+\beta_{b}\right) \angle \phi_{b} \\
\dot{I}_{c}=I_{a v}\left(1+\beta_{c}\right) \angle \phi_{c}
\end{array}\right.
$$

where, $I_{\mathrm{av}}$ is the mean value of three - phase current; $\beta_{A}, \beta_{B}$, $\beta_{C}$ is the unbalance of three phase current; $\phi_{a}, \phi_{b}, \phi_{c}$ is phase angle of three phase current.

For the three-phase four-wire system, when the three-phase unbalance occurs in the system, there is a current flowing through the neutral cable, and the current magnitude is:

$$
\begin{aligned}
\dot{I}_{U B, N L}=\dot{I}_{a}+\dot{I}_{b}+\dot{I}_{c} \\
=\left[\left(1+\beta_{a}\right) \cos \phi_{a}+\left(1+\beta_{b}\right) \cos \phi_{b}+\left(1+\beta_{c}\right) \cos \phi_{c}\right] I_{a v}+ \\
\quad \mathrm{j}\left[\left(1+\beta_{a}\right) \sin \phi_{a}+\left(1+\beta_{b}\right) \sin \phi_{b}+\left(1+\beta_{c}\right) \sin \phi_{c}\right] I_{a v}
\end{aligned}
$$

Define: 


$$
\left\{\begin{array}{c}
a=\left(1+\beta_{a}\right) \cos \phi_{a}+\left(1+\beta_{b}\right) \cos \phi_{b}+\left(1+\beta_{c}\right) \cos \phi_{c} \\
b=\left(1+\beta_{a}\right) \sin \phi_{a}+\left(1+\beta_{b}\right) \sin \phi_{b}+\left(1+\beta_{c}\right) \sin \phi_{c}
\end{array}\right.
$$

Then the current flowing through the cable neutral cable can be written as:

$$
\dot{I}_{U B, N L}=(a+j b) I_{a v}
$$

$$
\begin{aligned}
\Delta P_{U B, L} & =P_{U B, P L}+P_{U B, N L}-P_{B, L} \\
& =\left[\left(\beta_{a}^{2}+\beta_{b}^{2}+\beta_{c}^{2}+3\right) I_{a v}^{2}-3 I_{B, P L}^{2}\right] R_{F, P L}+\left(a^{2}+b^{2}\right) I_{a v}^{2} R_{F, N L}
\end{aligned}
$$

Similarly, additional losses on transformers under three-phase unbalance:

$$
\begin{gathered}
\Delta P_{U B, T}=P_{U B, T}-P_{B, T} \\
=\left[\left(\beta_{T a}^{2}+\beta_{T b}^{2}+\beta_{T c}^{2}+3\right) I_{T a v}^{2}-3 I_{B, T}^{2}\right] R_{F, T} \\
\Delta P_{L}=P_{F, L}+P_{H, L} \\
=\left[\left(\beta_{1 a}^{2}+\beta_{1 b}^{2}+\beta_{1 c}^{2}+3\right) I_{1 a v}^{2}-3 I_{B, y}\right. \\
\sum_{h=2}^{\infty}\left[\left(\beta_{h a}^{2}+\beta_{h b}^{2}+\beta_{h c}^{2}+3\right) I_{h a v}^{2}\right. \\
=\sum_{l=1}^{\infty}\left[\left(\beta_{l a}^{2}+\beta_{l b}^{2}+\beta_{l c}^{2}+3\right) I_{l a v}^{2} R_{l, P}\right. \\
\text { where } R_{h, P L} \text { as well as } R_{h, N L}, \text { is the harmonic resistance on } \\
\text { cable and neutral cable under combined harmonic and } \\
\text { three-phase unbalanced disturbances. } \\
\Delta P_{T}=\left[\left(\beta_{T 1 a}^{2}+\beta_{T 1 b}^{2}+\beta_{T 1 c}^{2}+3\right) I_{T 1 a v}^{2}-3 I_{B, T}^{2}\right] R_{1, T} \\
+\sum_{h=2}^{\infty}\left(\beta_{T h a}^{2}+\beta_{T h b}^{2}+\beta_{T h c}^{2}+3\right) I_{\text {Thav }}^{2} R_{h, T} \\
=\sum_{h=1}^{\infty}\left(\beta_{T h a}^{2}+\beta_{T h b}^{2}+\beta_{T h c}^{2}+3\right) I_{\text {Thav }}^{2} R_{h, T}-3 I_{B, T}^{2} R_{1, T}
\end{gathered}
$$$$
=\left[\left(\beta_{1 a}^{2}+\beta_{1 b}^{2}+\beta_{1 c}^{2}+3\right) I_{1 a v}^{2}-3 I_{B, P L}^{2}\right] R_{F, P L}+\left(a^{2}+b^{2}\right) I_{1 a v}^{2} R_{F, N L}+
$$$$
\sum_{h=2}^{\infty}\left[\left(\beta_{h a}^{2}+\beta_{h b}^{2}+\beta_{h c}^{2}+3\right) I_{h a v}^{2} R_{h, P L}+\left(a_{h}^{2}+b_{h}^{2}\right) I_{h a v}^{2} R_{h, N L}\right]
$$$$
=\sum_{l=1}^{\infty}\left[\left(\beta_{l a}^{2}+\beta_{l b}^{2}+\beta_{l c}^{2}+3\right) I_{l a v}^{2} R_{l, P L}+\left(a_{l}^{2}+b_{l}^{2}\right) I_{l a v}^{2} R_{l, N L}\right]-3 I_{B, P L}^{2} R_{F, P L}
$$

where $R_{h, T}$ is the harmonic resistance of transformer under combined harmonic and three-phase unbalanced disturbances.

\subsection{Simulation Verification Analysis}

According to the model and calculation method proposed in
As the fundamental loss and harmonic loss accord with the superposition, the cable and transformer losses are superimposed according to the fundamental loss and harmonic loss, which is:
The loss on the neutral cable is:

$$
P_{U B, P L}=I_{U B, N L}^{2} R_{F, N L}=\left(a^{2}+b^{2}\right) I_{a v}^{2} R_{F, P L}
$$

where $R_{F, N L}$ is the resistance of neutral cable under fundamental wave.

In summary, the additional loss of the cable in the case of three-phase unbalance is: this paper, firstly, the simulation verification is carried out on the condition of changing the three-phase unbalance degree based on the fixed injection harmonic current value. When the third and fifth harmonic currents in the distribution network remain unchanged at $2 \mathrm{~A}$, the additional losses of the cables and transformers are recorded according to Table 2 for changing the three-phase imbalance of loads. At the same time, according to the harmonic resistance calculation method proposed in this paper, the additional losses of cables and transformers under various conditions are calculated, and finally the results are compared and analyzed. The results are shown in Table 3, Figure 6 and Figure 7. In table $3, \mathrm{P}_{\mathrm{T}}$ is the loss of the transformer of the simulated result and $\mathrm{P}_{\mathrm{L}}$ is the loss of the cable of the simulated result with different three-phase load distributions. $\Delta \mathrm{P}$ is the total additional loss under combined power quality disturbances.

Table 2. Three-phase load distribution.

\begin{tabular}{llll}
\hline Three-phase load distribution & Phase A load/kVA & Phase B load /kVA & Phase C load /kVA \\
\hline Three-phase equilibrium & 20 & 20 & 20 \\
& 21 & 20 & 19 \\
Case 1 & 22 & 20 & 18 \\
& 23 & 20 & 22 \\
Case 2 & 19 & 19 & 24 \\
& 18 & 18 & 26 \\
Case 3 & 17 & 17 & 18 \\
& 21 & 21 & 16 \\
\hline
\end{tabular}




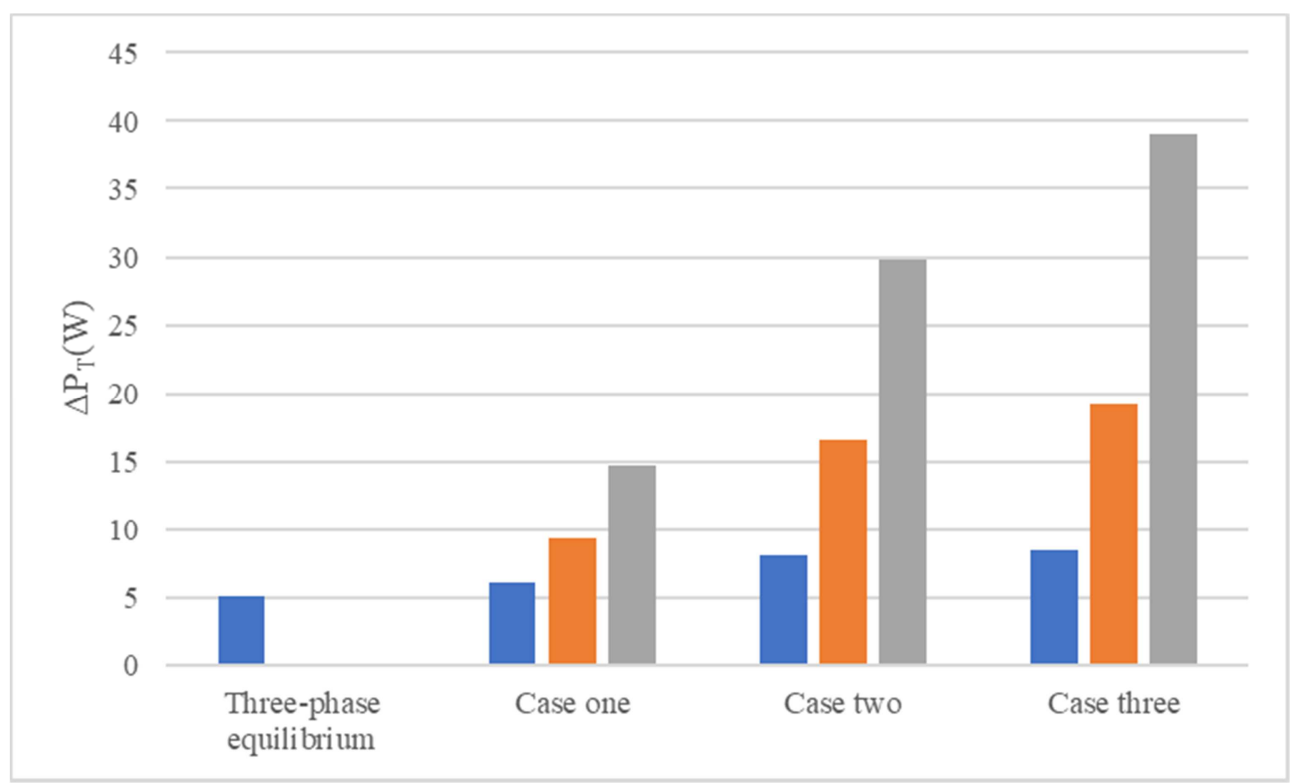

Figure 6. Additional loss of transformer under the disturbance of composite power quality changes with the distribution of three-phase load.

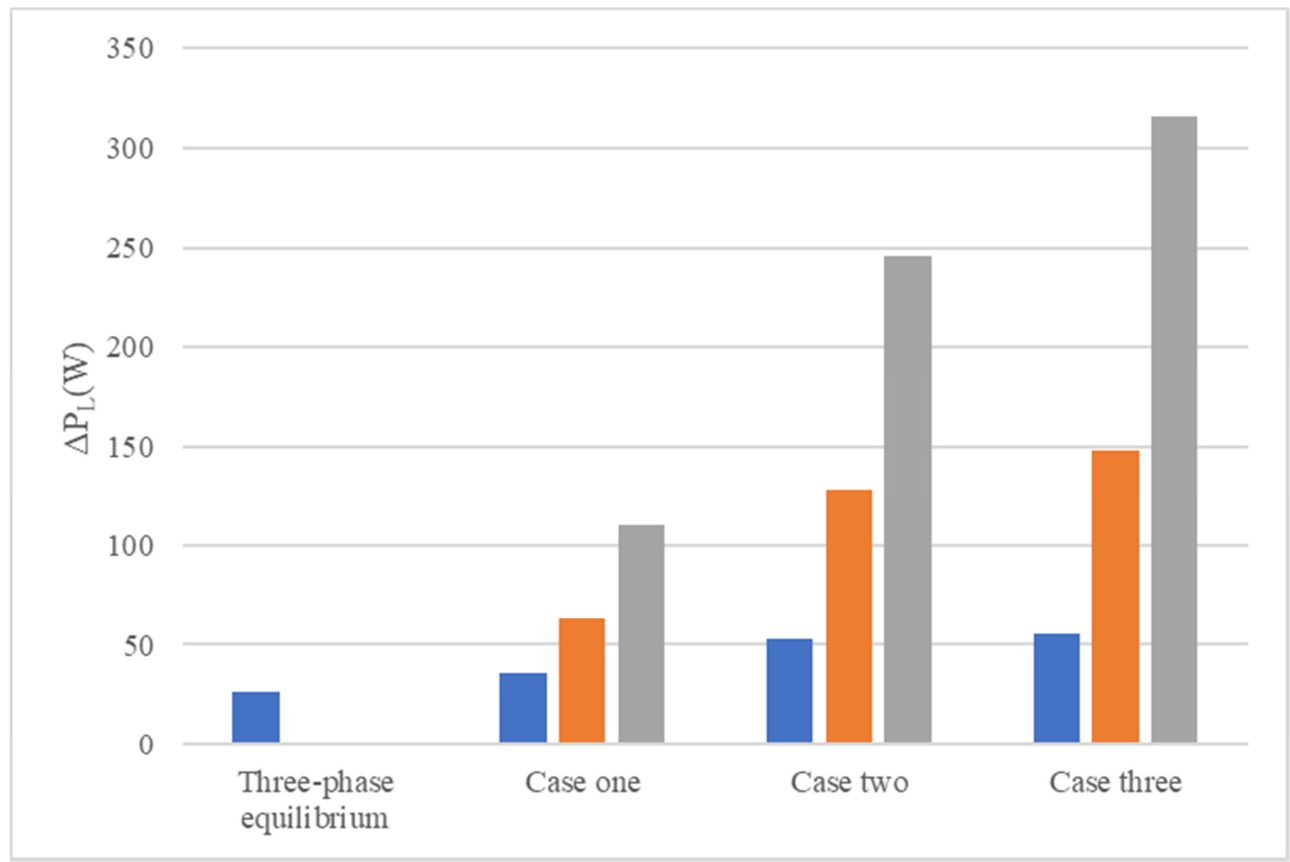

Figure 7. Additional cable losses under combined power quality disturbances with different three-phase load distributions.

Table 3. Distribution network loss analysis under different compound disturbances.

\begin{tabular}{llllll}
\hline A Phase imbalance & B Phase imbalance & $\mathbf{C}$ Phase imbalance & $\mathbf{P}_{\mathbf{T}} / \mathbf{W}$ & $\mathbf{P}_{\mathbf{L}} / \mathbf{W}$ & $\Delta \mathbf{P} / \mathbf{W}$ \\
\hline 0 & 0 & 0 & 5.052 & 26.501 & 31.553 \\
0.0251 & 0.0004 & -0.0255 & 6.110 & 35.831 & 41.942 \\
0.0500 & 0.0017 & -0.0517 & 9.304 & 63.490 & 72.793 \\
0.0747 & 0.0037 & -0.0786 & 14.683 & 109.974 & 124.656 \\
-0.0248 & -0.0245 & 0.0493 & 8.064 & 53.021 & 61.086 \\
-0.0485 & -0.0485 & 0.0966 & 16.550 & 128.160 & 144.710 \\
-0.0716 & -0.0710 & 0.1430 & 29.825 & 245.945 & 275.770 \\
0.0263 & 0.0259 & -0.0522 & 8.398 & 55.495 & 63.893 \\
0.0546 & 0.0546 & -0.1080 & 19.248 & 148.155 & 167.403 \\
0.0856 & 0.0838 & -0.1690 & 39.088 & 315.997 & 355.086 \\
\hline
\end{tabular}

According to Table 3, when the three-phase unbalance of the proposed method still has high accuracy. At the same time, the load is changed by keeping the harmonic current constant, it can be deduced that under the same conditions, the 
additional loss of distribution network caused by case 2 and case 3 is greater than that caused by case 1 , and this difference becomes more obvious with the increase of imbalance degree.

Similarly, keeping the three-phase unbalance of load unchanged in three cases, changing the third harmonic and fifth harmonic current in distribution network in turn, recording the results and comparative analysis. The results are shown in Figures 8 and 9 below.

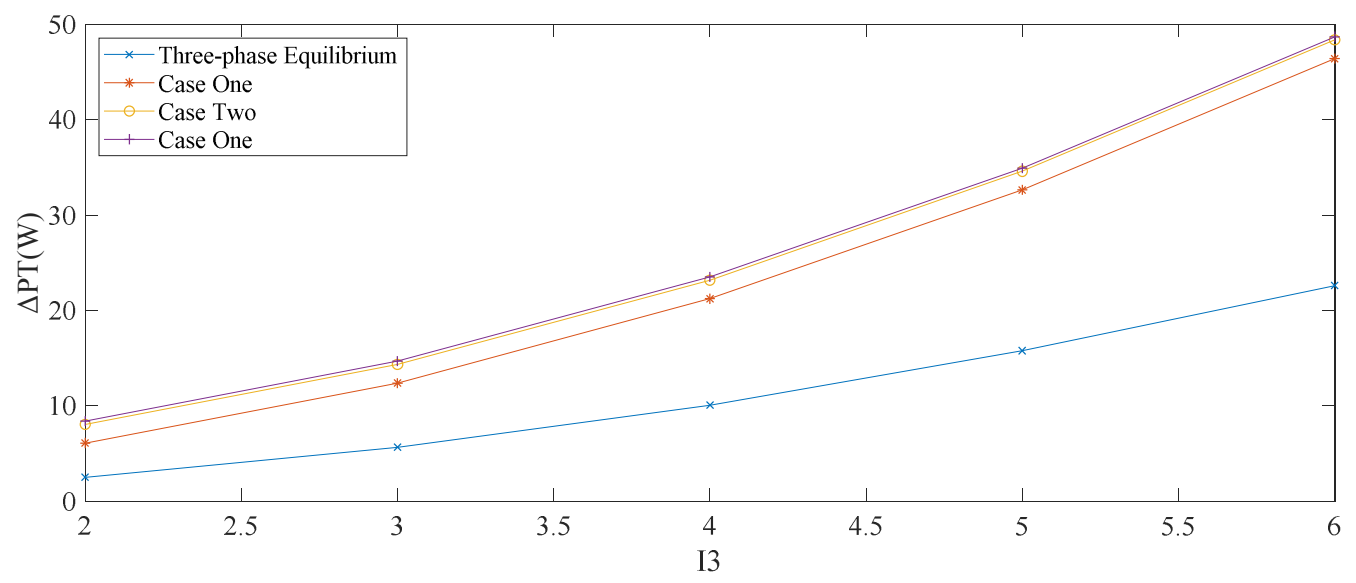

Figure 8. Analysis of transformer additional loss with the change of harmonic current under combined disturbance.

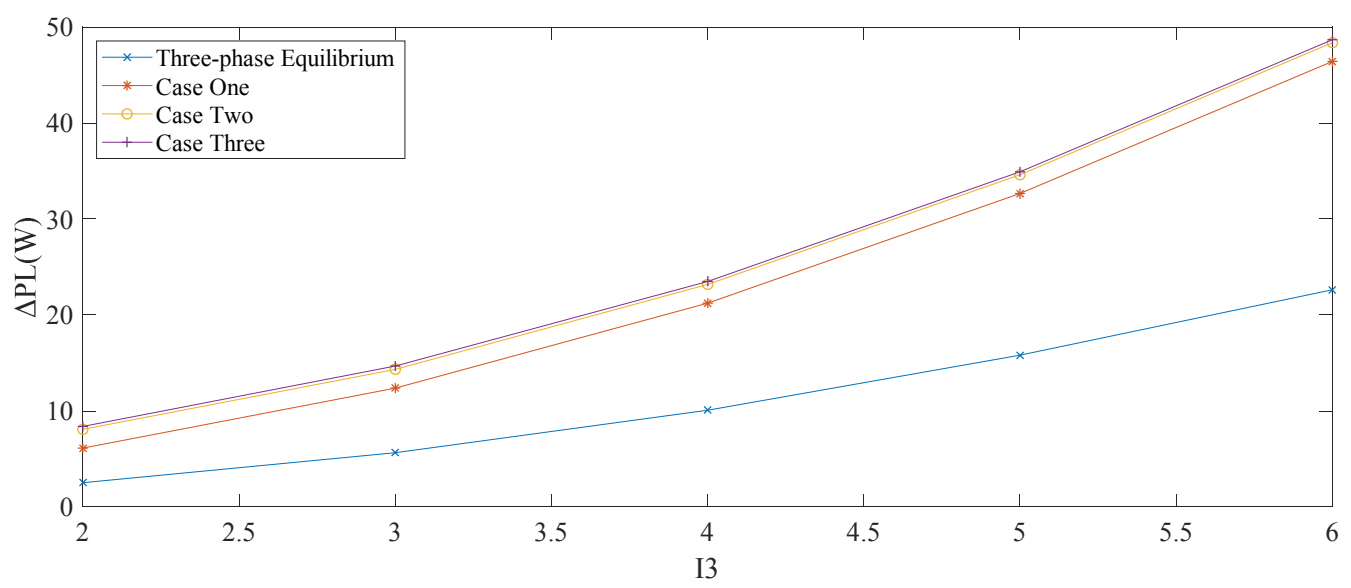

Figure 9. Analysis of cable additional loss with the change of harmonic current under combined disturbance.

According to the above charts, it is verified that the proposed method has high reliability under various complex composite power quality disturbances.

\section{Conclusion}

Aiming at the problem that a large number of researches on power quality do not fully consider the influence of skin effect on harmonic resistance, we propose a new calculation method of harmonic resistance on the basis of fully considering the influence of skin effect, and verify the accuracy of this method under harmonic disturbance and composite disturbance. According to the research based on the method in this paper, we can conclude that the harmonic resistance calculation method considering skin effect is more accurate. So the skin effect can not be ignored when we want to calculate the harmonic resistance. At the same time, when the harmonic current changes linearly, the additional loss generated increases nonlinearly. Finally, the influence of composite disturbance on the additional loss of the system is studied. Compared with other cases, when one phase load is heavy, one phase load is light, and one phase load is the average of the others, the additional loss of the system is the least affected. The additional loss for the case with that two phase load are heavy and one phase load is light is the biggest one. In summary, the proposed method has practical value and is helpful to further expand the influence of composite power quality disturbance on distribution network loss.

\section{Acknowledgements}

This work was supported by 'the Fundamental Research Funds for the Central Universities' (2018MS003).

\section{References}

[1] Wu Zhili, Wang Xuyang. Reflections on the implementation of the 13th five-year plan for distribution network [J]. Electric Power, 2017, 50 (1), pp. 25-29.

[2] Feng Cheng, Xu Changbao, Li Huaiyu, et al. Analysis of three phase current unbalance and harmonic influence on power loss. Electrical Applications, 2016, 35 (13), pp. 47-52. 
[3] Tao Yongwei, Zhcj Yong, Li Zequn. Calculation and analysis of distribution network loss considering three-phase unbalance. Information Technology, 2019 (7), pp. 82-85.

[4] Yin Zhongdong, Zhang Jing. Study on loss of key equipment in power distribution network under composite power quality disturbance. Electrical Applications, 2019, 38 (3), pp. 12-20.

[5] Xie Rongbin, Du Fan, Cheng Xiang, Zhou Qun, Xu Fangwei. Influence of three-phase imbalance and harmonic on line loss of three-phase four-wire low-voltage distribution network. Power system protection and control, 2020, 48 (21), pp. 22-30.

[6] Tofol IFL, Sanhueza S M R, Deoliveira A. On the study of losses in cables and transformers in nonsinusoidal conditions. IEEE Transact ions on Power Delivery, 2006, 21 (2), pp. 971-978.

[7] Miron A, Chindris M, Cziker A. Impact of unbalance in harmonic polluted power networks // International Symposium on Power Electronics. IEEE, 2012.

[8] Zhang Wenjie, Gandhi Oktoviano, HaoQuan, Rodríguez-Gallegos Carlos D, Srinivasan Dipti. A multi-agent based integrated volt-var optimization engine for fast vehicle-to-grid reactive power dispatch and electric vehicle coordination. Appl Energy 2018, 299, pp. 96-110.

[9] A. Chidurala, T. K. Saha, N. Mithulananthan, Harmonic impact of high penetration photovoltaic system on unbalanced distribution networks learning from an urban photovoltaic network, IET Renew. Power Gen. 10, 2016, pp. 485-494.
[10] R. Senra, W. C. Boaventura, E. M. A. M. Mendes, Assessment of the harmonic currents generated by single-phase nonlinear loads, Electr. Power Syst. Res. 147, 2017, pp. 272-279.

[11] S. Sakar, M. E. Balci, S. H. E. Abdel Aleem, A. F. Zobaa, Increasing PV hosting capacity in distorted distribution systems using passive harmonic filtering, Electr. Power Syst. Res. 148, 2017, pp. 74-86.

[12] M. M. Elkholy, M. A. El-Hameed, A. A. El-Fergany, Harmonic analysis of hybrid renewable microgrids comprising optimal design of passive filters and uncertainties, Electr. Power Syst. Res. 163, 2018, pp. 491-501.

[13] Dong Yunxia. Research on Loss in Power Grid Induced by Three-Phase Imbalance. Journal of Electrical and Electronic Engineering. 2020, (3), pp. 103-108.

[14] Bo Xing, Wang Zheng, Wu Yanbin. A new calculation method of distribution network line loss considering skin effect. Shanxi Electric Power, 2012 (03), pp. 38-41.

[15] A. A. Moustafa, A. M. Moussa, M. A. El-Gammal, "Separation of Customer and Supply Harmonics in Electrical Power Distribution Systems", Ninth International Conference on Harmonics and Quality of Power, 2000, Proceedings, (3), pp. 1035-1040.

[16] Mohammad A. S. Masoum, Ewald F. Fuchs, Harmonic Models of Transformers. Power Quality in Power Systems and Electrical Machines, 2008, pp. 55-108. 